\title{
NOTE ON COLD-JUNCTION CORRECTIONS FOR THERMOCOUPLES
}

\author{
By Paul D. Foote
}

Ordinarily, in the laboratory use of thermocouples, the cold junction is kept at a standard reference temperature such as the melting point of ice, while the hot junction is placed within the furnace or substance the temperature of which is desired. However, for technical purposes and general commercial use, it is not always convenient to have an ice box at hand. For this reason it is desirable to work with the cold junction at the temperature of the room. This necessitates a knowledge of the corrections to apply to the indicator readings, provided that the standardization of the pyrometer were carried out with the cold junction at some temperature other than that used in the technical works. The importance of these corrections is realized when one considers the large range of the cold-junction temperature which in practice frequently varies from 10 to $100^{\circ} \mathrm{C}$.

In order to obtain the temperature corresponding to any value of the emf observed when the cold junction is at a temperature $t_{0}{ }^{\circ} C$ from the relation $e=f(t)$ computed on the basis that the cold junction is at $o^{\circ} \mathrm{C}$, it is only necessary to add to the observed emf the value of the emf developed when the cold junction is at $o^{\circ} \mathrm{C}$ and the hot junction is $t_{o}^{\circ} C$, a number which is independent of the temperature of the furnace. However, many pyrometer galvanometers are calibrated to read temperature directly and have no scale of emf recorded. One must then express the correction in terms of degrees, and this quantity for any given value of the cold junction will depend, of course, upon the temperature of the hot junction for the reason that in general the relation between temperature and emf is not linear. A table, accordingly, might be prepared stating the corrections to be applied to the temperature observations for all values of the hot-junction temperature and all variations in the temperature of the cold junction. 
The usual method of obtaining the cold-junction correction consists in multiplying the value of the cold-junction temperature by certain empirical factors. $\mathrm{R}$. Vogel ${ }^{1}$ computed these factors for a Heraeus $(P t, 9 \circ P t-I o R h)$ couple having a cold junction of $50^{\circ} \mathrm{C}$. Offerhaus and Fischer ${ }^{2}$ have calculated the factors for several types of couples.

This method of correction for cold-junction temperatures may be derived in the following manner:

Derivation of the Slope Correction.-Let $e$ and $t$ be the observed emf and corresponding temperature when the cold junction $=0^{\circ} \mathrm{C}$.

$e_{1}$ and $t_{1}=$ the observed emf and temperature when the cold junction $=t_{o}^{\circ} C$.

For any thermocouple $\quad e=f(t)$

then for any value $e_{1} \quad e_{1}=f\left(t_{1}\right)$

But $\quad e=e_{1}+e_{0}$ where $e_{o}=f\left(t_{o}\right)=$ emf developed

when the hot junction $=t_{o}^{\circ}$ and the cold junction $=o^{\circ} \mathrm{C}$.

Substituting in (I) $\quad e_{1}+e_{o}=f(t)$

From (3)

From (2)

$$
\begin{gathered}
i=\varphi\left(e_{1}+e_{0}\right) \\
t_{1}=\varphi\left(e_{1}\right)
\end{gathered}
$$

Denote by $p$ the correction to add to the observed temperature when this value is read from the plot of a couple calibrated with its cold junction $=0^{\circ} \mathrm{C}$.

$$
\begin{aligned}
p=t-t_{1}= & \boldsymbol{\varphi}\left(e_{1}+e_{0}\right)-\boldsymbol{\varphi}\left(e_{1}\right) \\
= & \boldsymbol{\varphi}\left(e_{1}\right)+e_{o} \boldsymbol{\phi}^{\prime}\left(e_{1}\right)+\frac{e_{o}^{2}}{\frac{2}{2}} \boldsymbol{\phi}^{\prime \prime}\left(e_{1}\right)+\ldots-\boldsymbol{\varphi}\left(e_{1}\right) \\
= & e_{0} \boldsymbol{\phi}^{\prime}\left(e_{1}\right) \text { provided } e_{o} \text { and } t_{o} \text { are small in comparison } \\
& \text { with } e_{1} \text { and } t_{1}
\end{aligned}
$$

hence

$$
\begin{aligned}
& p=\frac{e_{o}}{\boldsymbol{\phi}\left(e_{0}\right)} \boldsymbol{\phi}^{\prime}\left(\mathrm{e}_{1}\right) \boldsymbol{\phi}\left(e_{o}\right)=\frac{e_{0}}{\boldsymbol{\phi}\left(e_{o}\right)} \cdot \frac{d t_{1}}{d e_{1}} \cdot t_{o} \\
& =\frac{d e_{0}}{d t_{o}} \quad \frac{d t_{1}}{d e_{1}} \quad t_{0}=\frac{\left(\frac{d e}{d t}\right)_{o}}{\left(\frac{d e}{d t}\right)_{1}} t_{0}
\end{aligned}
$$

approximately.

I R. Vogel: Zs. f. Anorg Chem., p. 45; 1905.

2 Cornelis Offerhaus and E. H. Fischer: Electrochem. and Metal. Ind., Sept., p. 362-364; 1908. 
This equation therefore states that in order to obtain the true temperature $(t)$, it is necessary to add to the observed temperature $\left(t_{1}\right)$, the quantity obtained by multiplying the temperature of the cold junction $\left(t_{0}\right)$ by the ratio of the slopes of the calibration curve at the origin and at the temperature $\left(t_{1}\right)$. The derivative $\left(\frac{d e}{d t}\right)_{0}$ is but approximately equal to the quantity $\frac{e_{0}}{\phi\left(e_{0}\right)}$ and becomes less so, the farther $t_{o}$ departs from zero. As seen from Fig. $1, \frac{e_{o}}{\boldsymbol{\phi}\left(e_{0}\right)}$ is more exactly the mean slope of the calibration curve from $o$ to $\mathrm{t}_{0}{ }^{\circ} \mathrm{C}$. Using this value the single term slope correction becomes:

$$
p=\frac{\left(\frac{d e}{d t}\right)_{o}-t_{0}}{\left(\frac{d e}{d t}\right)_{1}} \cdot t_{0}
$$

The mean slope from $o$ to $t_{o}$ may be determined accurately enough by assuming some value of $t_{o}$ in the neighborhood of the actual value of the cold-junction temperature such as $30^{\circ} \mathrm{C}$, and computing the mean slope from $o^{\circ}$ to this point, i. e., the value of the emf observed when the cold junction is at $o^{\circ}$ and the hot junction $30^{\circ}$ divided by 30 . This form of the cold-junction correction requires but a single measurement of the emf at room temperature, whereas the usual form of correction practically necessitates a knowledge of the curve at several points in order to evaluate the slope at $o^{\circ} \mathrm{C}$. The three forms of the cold-junction temperature correction may be summarized thus:

Form I $\quad p=e_{0} \phi^{\prime}\left(e_{1}\right)+\frac{e_{0}^{2}}{\underline{\underline{2}} \phi^{\prime \prime}}\left(e_{1}\right)+\ldots \ldots \frac{e_{0}^{n}}{\underline{n}} \phi^{n}\left(e_{1}\right)+\ldots$

Form II $p=\frac{\left(\frac{d e}{d t}\right)_{0}}{\left(\frac{d e}{d t}\right)_{1}}$

Form III $p=\frac{\left(\frac{d e}{d t}\right)_{0-30}}{\left(\frac{d e}{d t}\right)_{2}}$ 
Form I is the correct expression of the cold-junction correction. Form II is the approximation in general use.

Form III is the approximation which should be given preference over Form II.

Magnitude of the Cold-Junction Correction.-Fig. 2 illustrates the magnitude of the true cold-junction corrections for $P_{\mathbf{3}}$ a $(P t$, $90 P t-10 R h)$ Heraeus couple having for its temperature-emf relation the equations:

$$
\begin{array}{lr}
e \times 10^{4}=56.04 t+0.05434 t^{2} & \text { o to } 100^{\circ} \mathrm{C} \text { and } \\
e \times 10^{4}=-2820+80.71 t+0.01694 t^{2} & 300 \text { to } 1500^{\circ} \mathrm{C}
\end{array}
$$

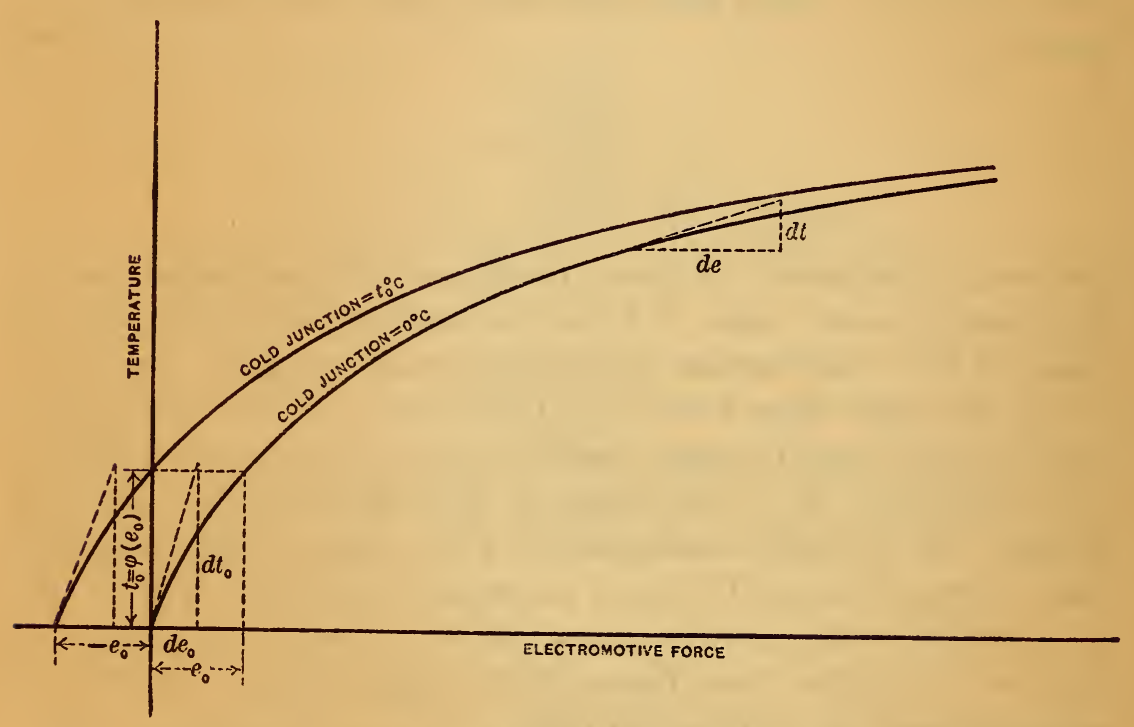

Fig. 1.-Illustrating the shift of the thermocouple curve with a change of the cold-junction temperature

where $e$ is expressed in millivolts. These curves represent the true correction, Form I, for four observed hot-junction temperatures, and with the cold-junction temperature varying from o to $100^{\circ} \mathrm{C}$. It is seen that the amount to be added to the observed temperature may reach a value as high as $60^{\circ}$ or $65^{\circ} \mathrm{C}$.

Errors in the Slope Corrections.-How closely Forms II and III express the true correction for the cold-junction temperature depends upon the form of the function $e=f(t)$. Inasmuch as the series in Form $I$ is frequently only slowly convergent a more 
convenient method than evaluating each term was used for determining the errors in the approximation corrections.

Several of the Bureau of Standards' thermocouples were calibrated (cold junction $=0^{\circ} \mathrm{C}$ ) from $o$ to $1500^{\circ} \mathrm{C}$. Assuming the temperature of the hot junction so regulated that any definite value of the observed emf developed by the couple remains constant whatever the temperature of the cold junction, the emf's for a cold junction $o^{\circ} \mathrm{C}$ were obtained by adding to the observed value, the emf readings between $\mathrm{o}$ and $100^{\circ} \mathrm{C}$. The temperatures

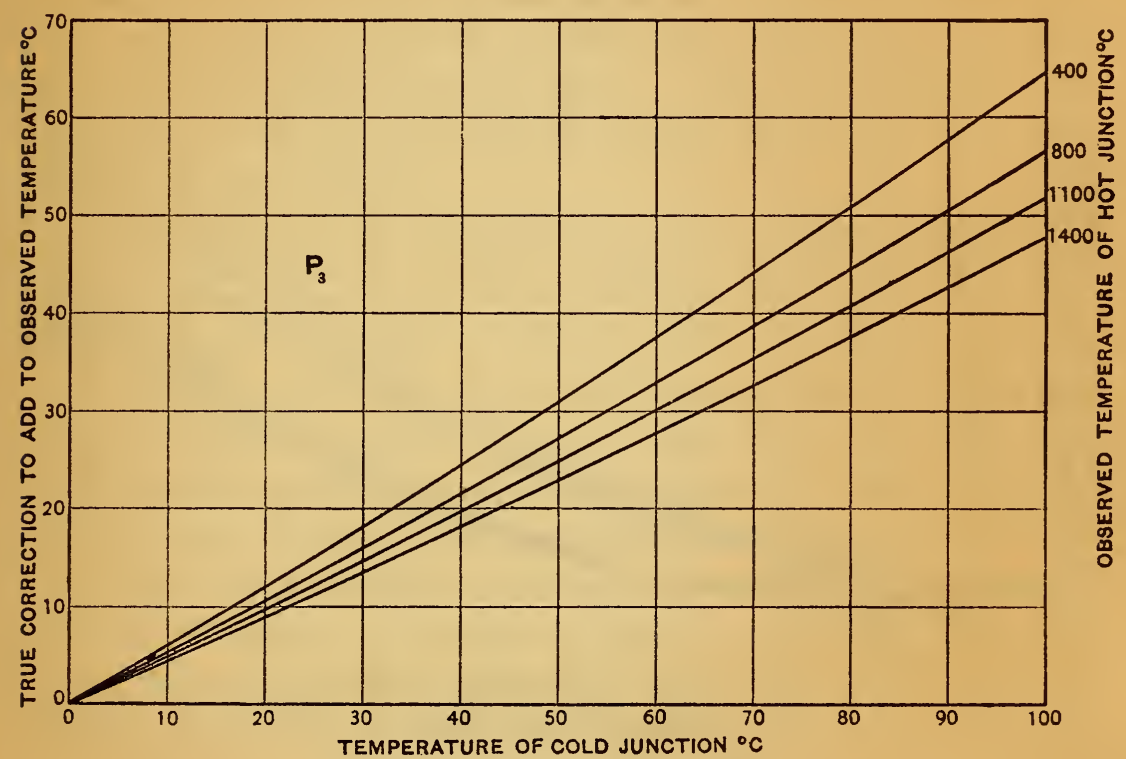

Fig. 2.-True correction $\nu$. cold-junction temperature

corresponding to these new enuf's were computed and from them was subtracted the temperature corresponding to the observed emf. The differences are the true corrections to be applied to the observed temperature for a series of cold-junction temperatures. This operation was repeated for several different observed values of the emf. The slope corrections were then computed for the same points.

Fig. 3 presents the errors met with in the application of the slope corrections, Forms II and III, to $P_{3}(P t, 90 P t-10 R h)$. The 
upper group of curves shows the amount to be added to the slope correction, Form II, to obtain the true correction. The largest error is $5^{\circ}$, with the hot junction at an observed temperature of $400^{\circ}$ and the cold junction $100^{\circ} \mathrm{C}$. The lower group of curves represents the corrections to apply when the slope correction is computed on the basis of the mean slope from o to $30^{\circ}$ instead of $0^{\circ} \mathrm{C}$, Form III. The errors are all somewhat smaller in this case, the maximum being $3^{\circ}$, and hence, in general, the correction according to Form III is to be preferred.

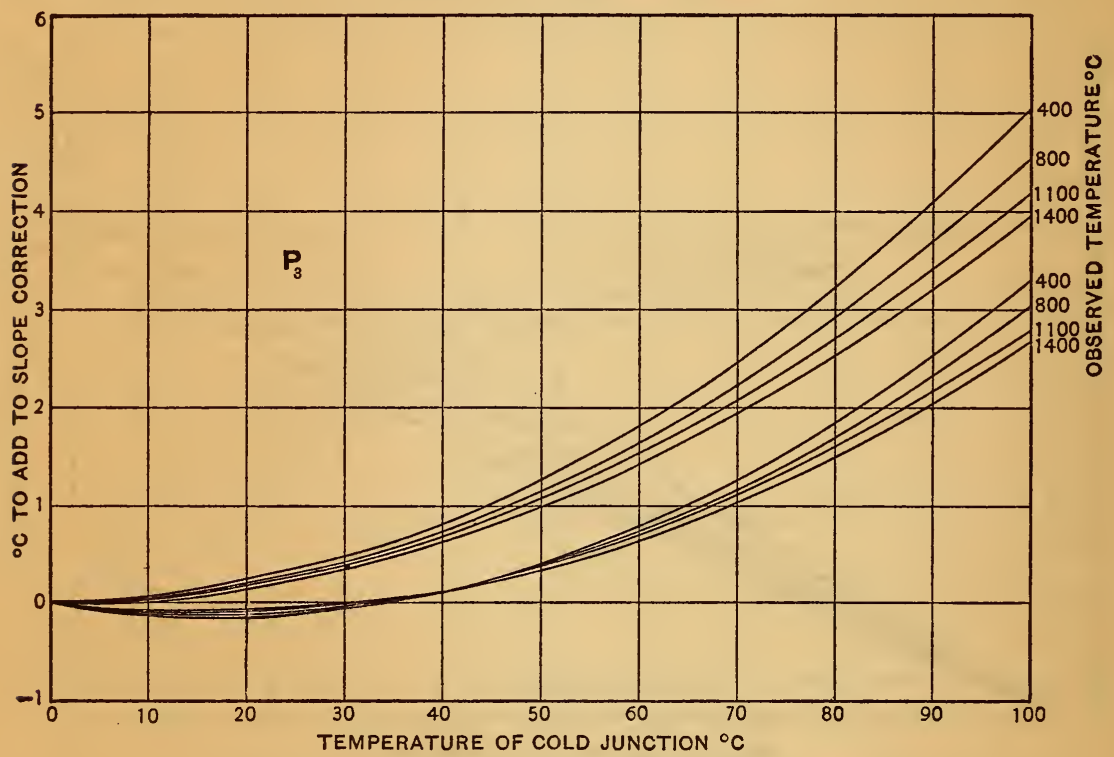

Fig. 3.-Error in slope correction

Similar curves were obtained for $P_{4}$, a Johnson and Matthey $(P t, 90 P t-10 R h)$ couple having the equations:
$e \times 1 \mathrm{I}^{4}=63.86 t+0.07343 t^{2}$
o to $100^{\circ} \mathrm{C}$
$e \times 10^{4}=-4934+99.06 t+0.01594 t^{2}$ 300 to $\mathrm{I}, 500^{\circ} \mathrm{C}$

Fig. 4 illustrates the errors found in the use of the slope corrections with $W_{7}$. This couple which was obtained from Pellin, and supposed to have the composition $(P t, 90 P t-\mathrm{Io} I r)$, had a temperature-emf relation expressed by the equations:
$e \times 10^{4}=84.76 t+0.1603 t^{2}$
o to $100^{\circ} \mathrm{C}$
$e \times 10^{4}=-6260+\mathrm{I} 30.2 t+0.0338 \mathrm{r} t^{2}$ 
The maximum error found for Form II is about $9^{\circ}$ with the hot junction at an apparent temperature of $400^{\circ}$ and the cold junction $100^{\circ} \mathrm{C}$. The lower group of curves is computed on the basis of the preferred form of the slope correction, Form III. The maximum error in this case is $6^{\circ}$.

For a copper-constantan couple of Adams and Johnston ${ }^{3}$ having the equation:

$e$ (microvolts) $=38.105 t+0.04442 t^{2}-0.00002856 t^{3}$ applicable over a range $o$ to $350^{\circ} \mathrm{C}$, the ordinary slope correction, Form II, is in error from $0.1^{\circ}$ to $0.5^{\circ}$ when the cold junction varies from o to $20^{\circ}$. With a cold junction of 20 to $50^{\circ}$ this slope correction is too small by amounts running from 0.5 to $2^{\circ}$. Form III applied to this couple shows corrections somewhat nearer those given by the true correction term, Form I.

Applicability of Slope Corrections.-If the couples examined are typical of all $(P t, 90 P t-10 R h),(P t, 90 P t-\mathrm{IoI} r)$, and copper-constantan couples, one may conclude that for values of the cold junction from $\mathrm{o}$ to $50^{\circ} \mathrm{C}$ in the case of the platinum-platinum alloy couples, and from o to $20^{\circ} \mathrm{C}$ in the case of the copper-constantan couple the slope correction, Form III, is fully applicable, and is not in error by amounts greater than the precision attainable with the ordinary pyrometric galvanometer. For all technical work, it is sufficient to add to the galvanometer temperature reading the number obtained by multiplying the ratio of the mean slope from o to $t_{0}$ and the slope at the observed temperature $\left(t_{1}\right)$ by the temperature $\left(t_{0}\right)$ of the cold junction.

The ratio of the slopes are given in the form of numerical factors for thermocouples tested at the Bureau of Standards, and to all technical purposes are alike for similar couples. Their determination requires only a rough temperature-emf calibration made independently of the pyrometer indicator. Instead of a table of figures, the observer need remember but two or three numbers applicable over all ranges of the cold-junction temperature. Thus in the use of $P_{3}$ with any type of galvanometer indicator, it is sufficient in ordinary pyrometric work to correct for the coldjunction temperature, $t_{0}^{\circ} \mathrm{C}$, by adding to the observed temperature,

${ }^{3}$ L. H. Adams and J. Johnston; Am. J. Sci., 33, p. 534, June, r9r2. 
$t_{1}^{\circ} \mathrm{C}$, the amount $0.6 t_{0}$ from 400 to $700^{\circ} \mathrm{C}$ and $0.5 t_{0}$ from 700 to

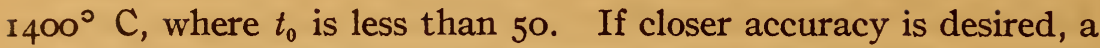
number of slope values computed to another significant figure, as given in Table I, second column, or preferably the third column, must be used, and the corresponding corrections shown in Fig. 3 applied. For the highest attainable accuracy a potentiometer is required, and the emf corresponding to the temperature of the cold junction is added to the emf reading of the couple.

TABLE 1

Temperature Centigrade vs. Cold-Junction Factor

$\mathbf{P}_{3}(\mathbf{P t}$, 90Pt-10Rh)

\begin{tabular}{c|c|c|c}
\hline Observed temperature & $\left(\frac{d e}{d t}\right) \div\left(\frac{d e}{d t}\right)_{1}$ & $\left(\frac{d e}{d t}\right)_{0-30} \div\left(\frac{d e}{d t}\right)_{1}$ & $\left(\frac{d e}{d t}\right)_{20-10} \div\left(\frac{d e}{d t}\right)_{2}$ \\
\hline $0 \mathrm{C}$ & 0.59 & 0.61 & 0.63 \\
400 & .57 & .59 & .61 \\
500 & .55 & .57 & .59 \\
600 & .53 & .55 & .57 \\
700 & .52 & .54 & .56 \\
800 & .50 & .52 & .54 \\
900 & .49 & .50 & .52 \\
1000 & .48 & .49 & .51 \\
1100 & .46 & .47 & .49 \\
1200 & .45 & .46 & .48 \\
1300 & .44 & .45 & .47 \\
1400 & &
\end{tabular}

The second column shows the factor by which the value of the cold-junction temperature must be multiplied when using the old-type slope correction, Form II. The third column contains the factors computed from the preferred form of the slope correction, Form III. The fourth column shows the factors when the couple is calibrated with the cold junction at $30^{\circ} \mathrm{C}$ and used with a cold-junction temperature not far from $40^{\circ} \mathrm{C}$.

Slope Correction when the Couple is Calibrated with the Cold Iunction at a Temperature other than $o^{\circ}$ C.- - Many thermocouple pyrometers are so constructed that it is impracticable to calibrate them with a cold junction at $0^{\circ} \mathrm{C}$. Suppose the standardization were carried out with a cold junction of $t_{0}=30^{\circ} \mathrm{C}$ and it is desired to use the couple near a furnace where the room temperature is ${ }_{2} t_{0}=40^{\circ} \mathrm{C}$. Proceeding by a method similar to that shown in the derivation of the ordinary slope correction, we now obtain the correction term:

$$
p=\frac{e_{0}}{{ }_{2} t_{0}-{ }_{1} t_{0}} \phi^{\prime}\left(e_{2}\right)\left({ }_{2} t_{0}-{ }_{1} t_{0}\right)
$$


where $\varphi^{\prime}\left(e_{2}\right)$ is the slope at the point corresponding to the observed emf, and $e_{0}=$ the emf developed when the cold junction is at the temperature ${ }_{1} t_{0}$ and the hot junction ${ }_{2} t_{0}$, or-

$$
p=\frac{\left(\frac{d e}{d t}\right)_{30-40}}{\left(\frac{d e}{d t}\right)_{2}}\left({ }_{2} t_{0}-30\right)
$$

where ${ }_{2} t_{0}$ is not far from $40, t_{2}$ being the observed temperature corresponding to $e_{2}$. This equation, therefore, states that in order to obtain the true temperature, $t$, it is necessary to add to the observed temperature, $t_{2}$, the quantity obtained by multiplying the difference in the temperature of the cold junction used in the standardization, ${ }_{1} t_{0}=30$, and during any observation, ${ }_{2} t_{0}=40$, by the ratio of the mean slope of the calibration curve from 30 to $40^{\circ}$ and the slope at the observed temperature. The mean slope from 30 to $40^{\circ}$ is obtained by dividing the emf observed when the cold junction is $30^{\circ}$ and the hot junction is $40^{\circ}$ by ro. The factors for $P_{3}$ used in this manner are given in Table I, column 4 .

Reduction to Fahrenheit Scale.-All the temperatures referred to above are given in degrees centigrade. The conversion to the Fahrenheit scale occasions no difficulty. When the couple is originally calibrated with the cold junction at the temperature of melting ice, $32^{\circ} \mathrm{F}$, and used with the cold junction at the temperature $t_{0}^{o} \mathrm{~F}$, the actual temperature, $t$, of the hot junction is obtained by adding to the observed temperature, $t_{1}$, the value of the product $\left(t_{0}-32\right) K$, instead of $t \cdot K$, where $K$ is the correction factor at the particular observed hot-junction temperature. If the couple is calibrated with the cold-junction temperature of ${ }_{1} t^{o}{ }_{0} \mathrm{~F}$ and used with the cold junction at a temperature ${ }_{2} t^{\circ}{ }_{0} \mathrm{~F}$., the correction term to be added to the observed temperature $t_{2}$ has the same form as before-

$$
p=\left({ }_{2} t_{0}-{ }_{1} t_{0}\right) K^{\prime}
$$

where $K^{\prime}$ is the factor computed from the mean slope between ${ }_{1} t_{o}$ and ${ }_{2} t_{0}$ and the slope at the observed temperature. The values of the ordinates for the curves in Figs. 2, 3, and 4 should be increased 
by the factor $9 / 5$, while the abscissas are directly converted to the Fahrenheit scale. As an example, in the case of Fig. 4 the ordinates will lie between the $o$ and 18 , the abscissas between 32 and $212^{\circ} \mathrm{F}$ and the observed temperatures of the hot junction become $752,1472,2012$, and $255^{\circ} \mathrm{F}$. In Table 2 are given the correction factors for $P_{3}(P t, 90 P t-10 R h)$ for a series of observed tempera-

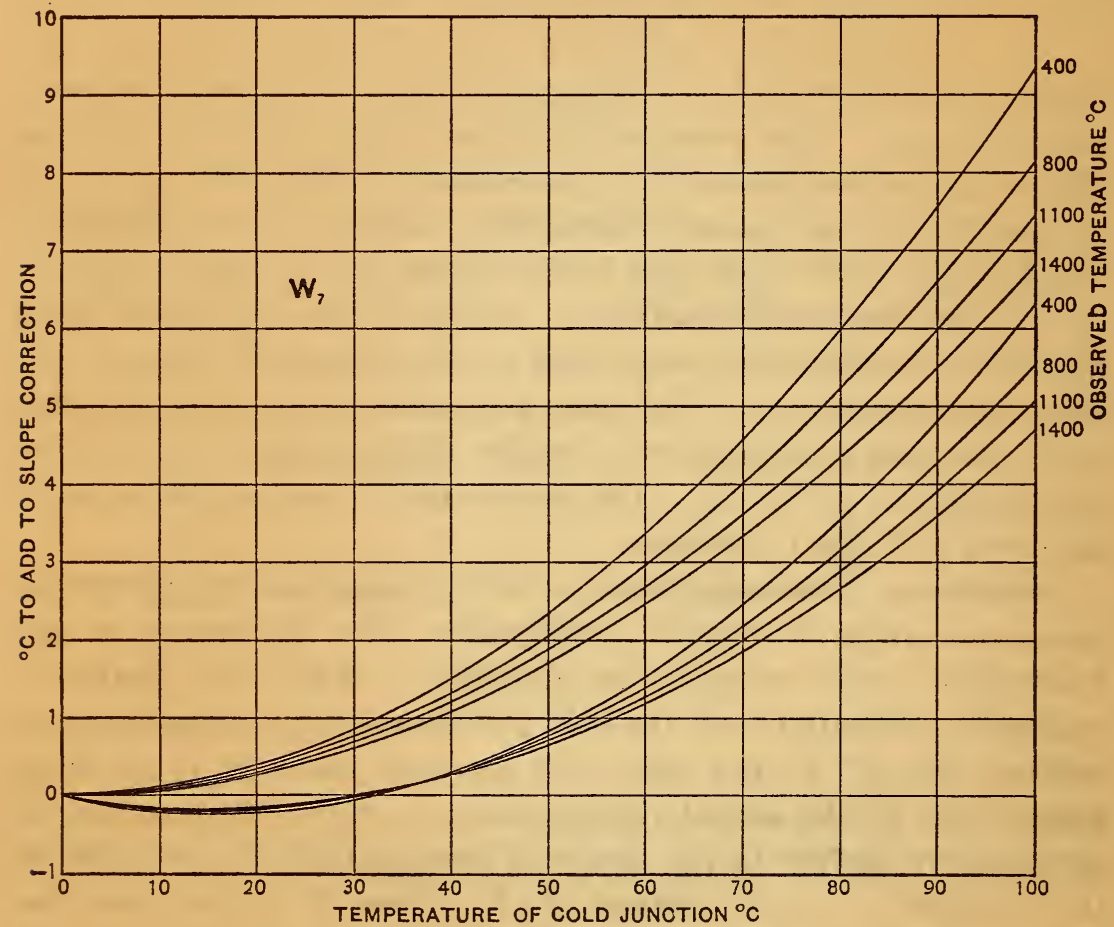

Fig. 4.-Error in slope correction

tures ${ }^{\circ} \mathrm{F}$. These follow directly from Table $\mathrm{I}$. The second column shows the factors computed on the basis of the old-slope correction, Form II. The third column contains the factors computed from the preferred slope correction, Form III. In the fourth column are given the correction factors when the couple is calibrated with a cold-junction temperature of $86^{\circ} \mathrm{F}$ and used with the cold junction not far from $104^{\circ} \mathrm{F}$. 
TABLE 2

Temperature Fahrenheit v. Cold-Junction Factor

$\mathbf{P}_{3}(\mathrm{Pt}, 90 \mathrm{Pt}-10 \mathrm{Rh})$

\begin{tabular}{c|c|c|c}
\hline Observed temperature. & $\left(\frac{d e}{d t}\right)_{32} \div\left(\frac{d e}{d t}\right)_{1}$ & $\left(\frac{d e}{d t}\right)_{32-66} \div\left(\frac{d e}{d t}\right)_{1}=K$ & $\left(\frac{d e}{d t}\right)_{86-104}\left(\frac{d e}{d t}\right)_{2}=K^{\prime}$ \\
\hline${ }^{\mathbf{F} .}$ & 0.59 & 0.61 & \\
800 & .56 & .58 & 0.63 \\
1000 & .54 & .56 & .60 \\
1200 & .53 & .54 & .58 \\
1400 & .51 & .52 & .56 \\
1600 & .49 & .51 & .54 \\
1800 & .48 & .49 & .53 \\
2000 & .46 & .47 & .51 \\
2200 & .44 & .45 & .49 \\
2400 & .43 & .44 & .48 \\
2600 & & .46 \\
\hline
\end{tabular}

Conversion of the Thermoelectric Scale to the Standard Temperature Scale.-When the ordinary parabolic equation $e=a+b t+c t^{2}$ is applied to a $(P t, 90 P t-10 R h)$ or $(P t$, 90 $P t$ - 1o $I r)$ thermocouple standardized at three temperatures, the melts of zinc, antimony, and copper, the curve extrapolated to $1500^{\circ} \mathrm{C}$ differs somewhat from the temperature shown by the gas thermometer. This is treated in full in The Measurement of High Temperatures, pages I I 2-1 I 6 loc. cit., but may be briefly summarized in the following table, which holds approximately for the usual type of platinum, platinum-iridium and platinum-rhodium couples.

TABLE 3

Correction to Thermocouple Scale

\begin{tabular}{c|c|c|c}
\hline Thermocouple scale. & $\begin{array}{c}\text { Correction to add degrees } \\
\text { Centigrade. }\end{array}$ & Thermocouple scale. & $\begin{array}{c}\text { Correction to add degrees } \\
\text { Fahrenheit }\end{array}$ \\
\hline${ }^{\circ} \mathrm{C}$. & & ${ }^{\circ}$ F. & \\
$300-1200$ & 0 & $600-2200$ & 0 \\
1300 & +2 & 2400 & +5 \\
1400 & +6 & 2600 & +14 \\
1500 & +14 & 2800 & +35 \\
\hline
\end{tabular}


The above discussion of couples $P_{3}, P_{4}$, and $W_{7}$ is on the basis of the thermoelectric scale. Hence, temperatures higher than $1200^{\circ} \mathrm{C}$ or $2200^{\circ} \mathrm{F}$ should be further corrected in accordance with Table 3. The order of applying the cold-junction correction and the above correction is of little importance, since both are small. Couples calibrated by the Bureau of Standards are corrected as shown in Table 3, so that the temperatures indicated by them accord with the established gas thermometer scale.

Devices for the Elimination of the Cold-Junction Corrections.Various methods have been proposed for the elimination of the cold-junction corrections. If a coil of copper wire were shunted across the terminals of the thermocouple in the head of the instrument, an increase in the temperature of the cold junction, ordinarily producing a decrease in the observed emf would increase the resistance of the shunt and thus tend to compensate for the thermoelectric effect. However, for exact compensation at all temperature ranges, a coil of wire would be required of which the resistance is a function not only of its own temperature, but also of the hot-junction temperature. This, of course, is impossible to realize in practice, but the method might be applied as a partial correction.

Bristol ${ }^{4}$ base metal couples are provided with extension wires which permit the location of the cold junction in a constant temperature room some distance from the furnace. Bristol has also devised an automatic compensator consisting of a bulb of mercury into which a loop of platinum wire dips. This is inserted in the circuit near the head of the couple so that the variations in the cold-junction temperature cause the mercury to expand or contract and short-circuit different lengths of the wire loop, thus altering the resistance with a result somewhat similar to that obtained by the use of a shunt coil.

Hartmann and Braun have the cold-junction head jacketed in order that a stream of water may be used to keep the head at a uniform temperature.

The Crompton Co. use a multiple scale on their galvonometers so constructed that the pointer reads directly the temperature of

1 The following and other methods of cold-junction compensation are discussed in The Measurement of High Temperatures, Burgess and Le Chatelier, p. 156, 3d ed., 1912, Wiley, New York. 
the furnace, each scale corresponding to some definite value of the cold-junction temperature.

Several instrument makers have tried interposing a supplementary thermocouple circuit of inexpensive material, the hot junction being located at the cold end of the main thermocouple and the cold junction at the galvanometer, some distance away.

Possibly the most simple compensating device is that employed by Siemens and Halske, and one or two other firms. Here the galvanometer indicator has a large zero adjustment so constructed that the pointer may be set, on open circuit, at the temperature corresponding to that of the cold junction, the graduations having been previously empirically determined for the given thermocouple.

All of these methods possess certain advantages but many of them are open to the objection that they are cumbersome and frequently liable to introduce new errors. Attention might well be given to the more careful construction of the indicating instruments. Many galvanometers have a temperature coefficient so large that small variations in the room temperature affect the readings to a marked degree. This may give rise to errors as large as those commonly found for the cold-junction temperature.

It is moreover needless to mention that the ordinary indicator should not be used in close proximity to other magnetic instruments or to iron unless originally calibrated in that position. A simple trial will readily convince one that this precaution is necessary.

When due care is exercised to locate the galvanometer in such a position that its magnetic field is not disturbed by outside influences and where its temperature is reasonably constant, to occasionally recheck the thermocouple calibration, and to properly correct for the cold-junction temperatures, the simple thermocouple without any compensating devices whatever will prove very satisfactory.

Acknowledgement is due Mr. I. N. Kellberg for computing and drawing the curves shown, and to Dr. G. K. Burgess for a number of suggestions.

Washington, April 15, 1913. 